\title{
Association between Pulse Pressure and Coronary Heart Disease in Korean Elderly: The 7th Korean National Health and Nutrition Examination Survey (2016-2018)
}

Yoojeong Lee(1), Geeyon Seo(1), Jiae Heo(1), Junggoo Kim(1), Hyojin Park(1), Jean Shin (1), Byoungduck Han (1), Joung Sik Son(1), Seon Mee Kim(1)

Department of Family medicine, Korea University Guro Hospital, Seoul, Korea

Background: Although the pulse pressure (PP) is an independent predictor of coronary heart disease (CHD), many physicians disregard its importance and there have been no recent studies on Korean elderly. We investigated the association between PP and CHD in Korean elderly through the nationwide, observational study.

Methods: This study included 4100 subjects aged $\geq 65$ years from the 7th Korean National Health and Nutrition Examination Survey (2016-2018). Pulse pressure was calculated as the difference between the systolic blood pressure (SBP) and the diastolic blood pressure (DBP), and high PP was defined as $\geq 60 \mathrm{mmHg}$. Subjects diagnosed with angina pectoris or myocardial infarction were classified into the CHD group. Multivariate logistic regression analysis was performed to evaluate the association between PP and CHD.

Results: Among the subjects, the incidence of high PP was higher in women, while CHD was higher in men. Subjects with high PP were older, had higher SBP, lower DBP and higher FBG compared to subjects with normal PP. After adjustment for multiple covariates, participants with high PP had a higher risk for incidence of CHD with an OR of 1.361 (95\% of CI: 1.077-1.719). Conclusions: In this study, we found the significant association between PP and CHD in Korean elderly.

Key Words: Coronary heart disease, Elderly, Pulse pressure, Risk factor

\section{INTRODUCTION}

Coronary heart disease (CHD) is one of the leading causes of death worldwide [1,2]. Although CHD mortality has declined gradually [3], the incidence of CHD has increased as lifespan extends $[4,5]$. Since the incidence of CHD itself causes socioeconomic burden, the prevention of $\mathrm{CHD}$ is important.
CHD is a multifactorial disease [6,7]. As it is well known, various factors contribute to $\mathrm{CHD}$ such as aging, hypertension (HTN), dyslipidemia and smoking. Pulse pressure (PP) is the difference between the systolic blood pressure (SBP) and diastolic blood pressure (DBP). As already confirmed in several studies including the Framingham study, $\mathrm{PP}$ is an independent predictor of CHD [8,9]. Also, the measurement of PP is a non-invasive and cost-effective tool

Received June 25, 2021; revised August 7, 2021; accepted August 24, 2021.

Corresponding author: Yoojeong Lee, Department of Family medicine, Korea University Guro Hospital, 148 Gurodong-ro, Seoul 08308, Korea. E-mail: juujeong@naver.com

Copyright (C) 2021 The Korean Academy of Clinical Geriatrics

() This is an open access article distributed under the terms of the Creative Commons Attribution Non-Commercial License (http://creativecommons.org/licenses/by-nc/4.0) which permits unrestricted non-commercial use, distribution, and reproduction in any medium, provided the original work is properly cited. 
to predict the incidence of CHD simply measured in primary care settings [10]. Despite the usefulness of measuring PP, there was no recent study on Korean elderly. Thus, this study was aimed to find the correlation between PP and CHD in Korean elderly through the nationwide, large-scale observational study.

\section{MATERIALS AND METHODS}

\section{Study subjects}

This study was based on data from the 7th Korean National Health and Nutrition Examination Survey (KNHANES VII) conducted for 3 years (2016-2018). KNHANES VII is a population-based, cross- sectional study performed by the Korea Centers for Disease Control and Prevention (KCDC). The survey consisted of three parts: a health interview survey, a health examination survey, and a nutrition survey. The number of participants in the survey was 24,269 . Among them, elderly aged over 65 years was 4,956. We excluded 856 participants with incomplete data such as height, weight, body mass index (BMI), waist circumference (WC), SBP, DBP readings, and blood chemistry test results. Finally, 4,100 subjects were analyzed in this study (Figure. 1). This study was conducted after obtaining approval for exemption from review by the Research Ethics Review Committee (Registration Number : K2021-0747-001).

\section{Patient's dermographic characteristics and clinical variables}

Data regarding demographics and clinical variables including sex, age, smoking habits, and alcohol consumption were obtained using a health questionnaire. Sex was classified into male and female. In the smoking category, only current smokers were classified as the smoking group. In the alcohol consumption category, according to the health questionnaire who consumed alcohol at least once a month over the last year were classified as alcohol consumption group. Comorbidities that can affect CHD such as diabetes mellitus (DM), dyslipidemia, and hypertension (HTN) were defined as those who were currently diagnosed by a physician.

Anthropometric data included measurement of weight, height, BMI, WC, SBP, and DBP. Weight, height and WC were measured following standardized protocols shown in KNHANES VII guidebook. Weight was measured using a GL-6000-20 (G-tech, Korea), height was measured with Seca 225 (Seca, Germany) or Seca 210 (Seca, Germany). BMI was calculated as weight in kilogram $(\mathrm{kg})$ divided by height in $\mathrm{m}^{2}$. WC was measured using a Seca 200 (Seca, Germany). Blood pressure was measured using a Baumanometer $\left.{ }^{(}\right)$Wall Unit 33 (0850) (Baum, USA), subjects with in a sitting position after taking 5-minute break. After three measurements, the average value of the 2 nd and 3 rd blood pressure was used as data.

Laboratory data included estimation of fasting blood glucose (FBG), total cholesterol (TC), high-density lipoproteincholesterol (HDL-C), low-density lipoproteincholesterol (LDL-C), and triglycerides (TG). Blood samples were obtained after fasting at least $8 \mathrm{hrs}$ with immediate pretreatment to minimize interference. The samples were analyzed by Labospect008AS (Hitachi/Japan) using enzymatic method and hexokinase UV.

\section{Definition of high PP and CHD}

$\mathrm{PP}$ was calculated as the difference between the SBP and DBP. High PP was defined as PP $\geq 60 \mathrm{mmHg}$ [11]. In the evaluation of $\mathrm{CHD}$, subjects with angina pectoris or my-

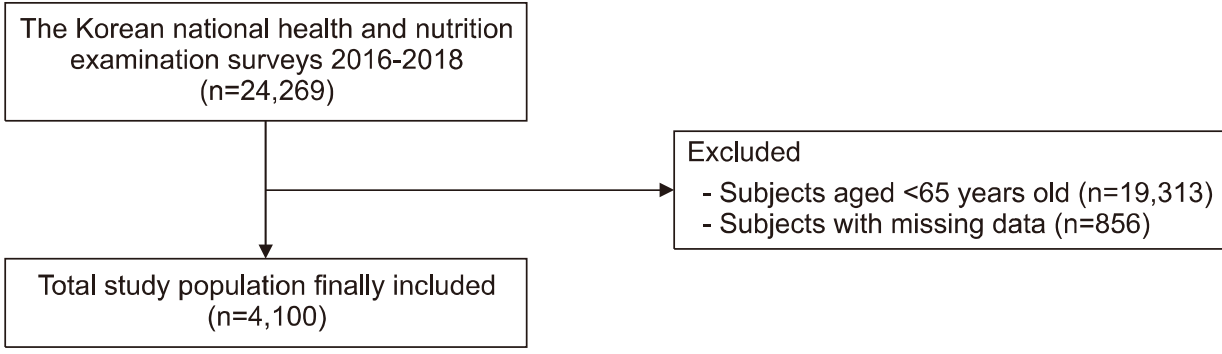

Figure 1. Flow diagram outlining the inclusion and exclusion criteria and the study design. 
ocardial infarction diagnosed by a physician were classified into the CHD group.

\section{Statistical analysis}

General characteristics of the study subjects according to sex and the PP were analyzed using a chi-square test (continuous variables) and an independent t-test (categorical variables), and the results were presented in terms of mean, standard deviation, and percentage. The association with PP to variables was estimated using Pearson correlation analysis. TG values were analyzed after converting these into log values. To investigate the effect of variables on CHD, we performed multivariate logistic regression analysis was performed with PP, age, sex, smoking, alcohol consumption, DM, serum HDL-C, and LDL-C levels as independent variables with the diagnosis of $\mathrm{CHD}$ as the dependent variable (Model 1: non-adjusted, Model 2: adjusted for age, sex,
Model 3: further adjusted for smoking habits and alcohol consumption, Model 4 : further adjusted for DM, HDL-C, and LDL-C).

All statistical analyses were performed using the SPSS Statistics version. 27. Results were presented as of odds ratio and $95 \%$ confidence interval. The level of statistical significance in each analysis was defined as $\mathrm{P}$-value $<0.05$.

\section{RESULTS}

\section{General characteristics of subjects}

General characteristics of the research subjects are shown in Table 1. The sample comprised 1,808 men (44.1\%) and 2,292 women (55.9\%). The mean age was $72.6 \pm 5.0$ and there was no significant difference between the two groups. Compared to male participants, the female participants had a higher BMI, higher SBP, higher PP (all $\mathrm{P}<0.001$ ). The

Table 1. General characteristics of research subjects $(N=4,100)$

\begin{tabular}{|c|c|c|c|c|}
\hline & Total & Men $(n=1808)$ & Women $(n=2292)$ & $\mathrm{P}$-value* \\
\hline Age & $72.6 \pm 5.0$ & $72.5 \pm 4.9$ & $72.8 \pm 5.1$ & 0.054 \\
\hline Weight (kg) & $60.5 \pm 9.8$ & $65.3 \pm 9.1$ & $56.8 \pm 8.6$ & $<0.001$ \\
\hline Height (cm) & $158.0 \pm 8.9$ & $165.6 \pm 5.7$ & $151.9 \pm 5.8$ & $<0.001$ \\
\hline BMI $\left(\mathrm{kg} / \mathrm{m}^{2}\right)$ & $24.2 \pm 3.2$ & $23.8 \pm 2.9$ & $24.6 \pm 3.3$ & $<0.001$ \\
\hline $\mathrm{WC}(\mathrm{cm})$ & $85.4 \pm 9.0$ & $86.9 \pm 8.7$ & $84.3 \pm 9.1$ & $<0.001$ \\
\hline SBP (mmHg) & $128.9 \pm 17.5$ & $126.7 \pm 16.7$ & $130.6 \pm 17.9$ & $<0.001$ \\
\hline DBP (mmHg) & $72.5 \pm 9.9$ & $72.2 \pm 9.8$ & $72.8 \pm 9.9$ & 0.066 \\
\hline $\mathrm{PP}(\mathrm{mmHg})$ & $56.3 \pm 15.2$ & $54.5 \pm 14.5$ & $57.8 \pm 15.6$ & $<0.001$ \\
\hline High $\mathrm{PP}(\mathrm{PP} \geq 60)$ & $1561(38.1)$ & 601 (33.2) & $960(41.9)$ & $<0.001$ \\
\hline $\mathrm{FBG}(\mathrm{mg} / \mathrm{dL})$ & $108.0 \pm 26.6$ & $109.8 \pm 28.4$ & $106.6 \pm 25.0$ & $<0.001$ \\
\hline $\mathrm{TC}(\mathrm{mg} / \mathrm{dL})$ & $184.5 \pm 39.7$ & $178.0 \pm 37.9$ & $189.6 \pm 40.4$ & $<0.001$ \\
\hline HDL-C (mg/dL) & $48.1 \pm 11.9$ & $46.1 \pm 11.7$ & $49.7 \pm 11.7$ & $<0.001$ \\
\hline LDL-C (mg/dL) & $109.5 \pm 36.4$ & $105.0 \pm 34.5$ & $113.1 \pm 37.5$ & $<0.001$ \\
\hline $\mathrm{TG}(\mathrm{mg} / \mathrm{dL})$ & $134.2 \pm 83.1$ & $134.5 \pm 80.5$ & $134.0 \pm 85.1$ & 0.865 \\
\hline Current smoking $^{+}$ & $377(9.2)$ & $327(18.1)$ & $50(2.2)$ & $<0.001$ \\
\hline Alcohol (yes) ${ }^{\ddagger}$ & $1,451 \quad(35.4)$ & $1,039(57.5)$ & $412(18.0)$ & $<0.001$ \\
\hline HTN $(\text { yes })^{\S}$ & $2,603(63.5)$ & $1,072(59.3)$ & $1,531(66.8)$ & $<0.001$ \\
\hline DM (yes $)^{\S}$ & $1,078(26.3)$ & $503(27.8)$ & $575(25.1)$ & 0.048 \\
\hline Dyslipidemia $(y e s)^{\S}$ & $1,390(33.9)$ & $446(24.7)$ & $944(41.2)$ & $<0.001$ \\
\hline $\mathrm{CHD}(\text { yes })^{\S}$ & $331(8.1)$ & $183(10.1)$ & $148(6.5)$ & $<0.001$ \\
\hline
\end{tabular}

Data are expressed as mean \pm standard deviation or $\mathrm{n}(\%)$.

BMI, body mass index; WC, waist circumference; SBP, systolic blood pressure; DBP, diastolic blood pressure; PP, pulse pressure; FBG, fasting blood glucose; TC, total cholesterol; HDL-C, high density lipoprotein-cholesterol; LDL-C, low density lipoprotein-cholesterol; TG, triglycerides; HTN, hypertension; DM, diabetes mellitus; CHD, coronary heart disease.

*P-value were obtained by chi-square test (continuous variable) and independent t-test (categorial variable). ${ }^{\dagger}$ Current smokers were classified as smoking group. ${ }^{\ddagger}$ Participants who consumed at least one glass of alcohol every month over the last year were classified as alcohol consumption group. ${ }^{\S}$ Comorbidities were defined as those who were currently diagnosed by doctor. 
incidence of high PP and CHD were 1561 (38.1\%), and 331 (8.1\%), respectively. The incidence of high $\mathrm{PP}$ was higher in women $(\mathrm{P}<0.001)$, while the $\mathrm{CHD}$ was higher in men. $(\mathrm{P}<0.001)$.

\section{General characteristics of subjects according to high PP}

General characteristics of the research subjects according to high PP are shown in Table 2. Individuals with high PP were older while they had higher SBP, lower DBP and higher FBG (all $\mathrm{P}<0.001$ ). The high $\mathrm{PP}$ group was less likely to consume alcohol $(\mathrm{P}=0.005)$. More subjects were diagnosed with hypertension $(\mathrm{P}<0.001)$, diabetes mellitus $(\mathrm{P}<0.001)$ and dyslipidemia $(\mathrm{P}=0.043)$ in the high $\mathrm{PP}$ group.

\section{Correlations between study variables and PP}

Table 3 shows the correlation between the investigated variables and the PP. In the Pearson correlation analysis showed that age, sex, FBG, and serum TG levels were associated with elevated in PP.

The serum LDL-C level was associated with reduced in PP.

\section{Comparison of the CHD odd ratios according to high pulse pressure}

Table 4 shows the OR and 95\% CIs of multivariates adjusted logistic regression between high $\mathrm{PP}$ and the incidence of CHD. There was significant association between high PP and CHD without adjustment (Model 1). After adjustment for age and sex, individuals with high PP had a higher risk for incidence of CHD with an OR of 1.36 (95\% of CI : 1.08-1.72). After additionally adjustment for serum HDL-C and LDL-C level and DM, the OR for CHD was 1.29 (95\% CI: 1.03-1.82) (Model 4).

Table 2. General characteristics of research subjects according to PP $(n=4,100)$

\begin{tabular}{|c|c|c|c|c|}
\hline & Total & Normal PP $(n=1808)$ & High PP $(n=2292)$ & P-value* \\
\hline Age & $72.6 \pm 5.0$ & $71.7 \pm 4.9$ & $74.1 \pm 4.8$ & $<0.001$ \\
\hline Weight $(\mathrm{kg})$ & $60.5 \pm 9.8$ & $60.5 \pm 9.8$ & $59.3 \pm 9.5$ & $<0.001$ \\
\hline Height $(\mathrm{cm})$ & $158.0 \pm 8.9$ & $159.0 \pm 9.0$ & $156.3 \pm 8.6$ & $<0.001$ \\
\hline $\operatorname{BMI}\left(\mathrm{kg} / \mathrm{m}^{2}\right)$ & $24.2 \pm 3.2$ & $24.2 \pm 3.2$ & $24.2 \pm 3.1$ & 0.791 \\
\hline $\mathrm{WC}(\mathrm{cm})$ & $85.4 \pm 9.0$ & $85.5 \pm 9.1$ & $85.4 \pm 9.0$ & 0.707 \\
\hline $\mathrm{SBP}(\mathrm{mmHg})$ & $128.9 \pm 17.5$ & $119.9 \pm 12.3$ & $143.4 \pm 14.8$ & $<0.001$ \\
\hline $\mathrm{DBP}(\mathrm{mmHg})$ & $72.5 \pm 9.9$ & $73.0 \pm 9.3$ & $71.8 \pm 10.7$ & $<0.001$ \\
\hline $\mathrm{FBG}(\mathrm{mg} / \mathrm{dL})$ & $108.0 \pm 26.6$ & $106.8 \pm 26.2$ & $109.9 \pm 27.1$ & $<0.001$ \\
\hline $\mathrm{TC}(\mathrm{mg} / \mathrm{dL})$ & $184.5 \pm 39.7$ & $184.5 \pm 39.7$ & $183.7 \pm 40.1$ & 0.302 \\
\hline $\mathrm{HDL}-\mathrm{C}(\mathrm{mg} / \mathrm{dL})$ & $48.1 \pm 11.9$ & $47.9 \pm 11.8$ & $48.4 \pm 11.9$ & 0.218 \\
\hline LDL-C (mg/dL) & $109.5 \pm 36.4$ & $110.4 \pm 36.4$ & $108.1 \pm 36.4$ & 0.041 \\
\hline $\mathrm{TG}(\mathrm{mg} / \mathrm{dL})$ & $134.2 \pm 83.1$ & $133.1 \pm 88.7$ & $136.1 \pm 73.1$ & 0.264 \\
\hline Current smoking $^{\dagger}$ & $377(9.2)$ & $243(9.6)$ & $134(8.6)$ & 0.289 \\
\hline Alcohol (yes) & $1,451(35.4)$ & $940(37.0)$ & $511(32.7)$ & 0.005 \\
\hline HTN (yes) $)^{\S}$ & $2,603(63.5)$ & $1,283(50.5)$ & $1,320(84.6)$ & $<0.001$ \\
\hline $\mathrm{DM}(\text { yes })^{\S}$ & $1,078(26.3)$ & $595(23.4)$ & $483(30.9)$ & $<0.001$ \\
\hline Dyslipidemia $(y e s)^{\S}$ & $1,390(33.9)$ & $831(32.7)$ & $559(35.8)$ & 0.043 \\
\hline $\mathrm{CHD}(\text { yes })^{\S}$ & $331(8.1)$ & $183(7.2)$ & $148(9.5)$ & 0.009 \\
\hline
\end{tabular}

Data are expressed as mean \pm standard deviation or $\mathrm{n}(\%)$.

BMI, body mass index; WC, waist circumference; SBP, systolic blood pressure; DBP, diastolic blood pressure; PP, pulse pressure; FBG, fasting blood glucose; TC, total cholesterol; HDL-C, high density lipoprotein-cholesterol; LDL-C, low density lipoprotein-cholesterol; TG, triglycerides; HTN, hypertension; DM, diabetes mellitus; CHD, coronary heart disease.

*P-value were obtained by chi-square test (continuous variable) and independent t-test (categorial variable). ${ }^{\dagger}$ Current smokers were classified as smoking group. ${ }^{\ddagger}$ Participants who consumed at least one glass of alcohol every month over the last year were classified as alcohol consumption group. ${ }^{\S}$ Comorbidities were defined as those who were currently diagnosed by doctor. 
Table 3. Correlations among observed variable and PP

\begin{tabular}{|c|c|c|c|}
\hline & & $\mathrm{PP}$ & $\mathrm{P}$-value \\
\hline \multirow[t]{9}{*}{ Pearson correlation coefficient* } & Sex & 0.107 & $<0.001$ \\
\hline & Age & 0.281 & $<0.001$ \\
\hline & $\operatorname{BMI}\left(\mathrm{kg} / \mathrm{m}^{2}\right)$ & 0.019 & 0.223 \\
\hline & $\mathrm{WC}(\mathrm{cm})$ & -0.001 & 0.971 \\
\hline & $\mathrm{FBG}(\mathrm{mg} / \mathrm{dL})$ & 0.062 & $<0.001$ \\
\hline & $\mathrm{TC}(\mathrm{mg} / \mathrm{dL})$ & -0.012 & 0.433 \\
\hline & HDL-C (mg/dL) & 0.020 & 0.193 \\
\hline & LDL-C (mg/dL) & -0.033 & 0.034 \\
\hline & $\mathrm{TG}(\mathrm{mg} / \mathrm{dL})$ & 0.057 & $<0.001$ \\
\hline
\end{tabular}

BMI, body mass index; WC, waist circumference; FBG, fasting blood glucose; TC, total cholesterol; HDL-C, high density lipoprotein-cholesterol; LDL-C, low density lipoprotein-cholesterol; TGs, triglycerides; PP, pulse pressure.

*Pearson correlation coefficient was obtained by Pearson correlation.

Table 4. Comparisons of $\mathrm{CHD}$ odds ratio according to high PP $(\mathrm{N}=4,100)$

\begin{tabular}{clcccr}
\hline \multirow{2}{*}{ Category } & & \multicolumn{5}{c}{ CHD odds ratio } \\
\cline { 3 - 6 } & & Model 1 & Model 2 & Model 3 & Model 4 \\
\hline \multirow{2}{*}{ PP } & Normal PP & 1 (Ref) & 1 (Ref) & 1 (Ref) & 1 (Ref) \\
& High PP & $1.35(1.08-1.69)$ & $1.35(1.06-1.70)$ & $1.35(1.06-1.70)$ & $1.29(1.03-1.82)$ \\
& P-value & 0.01 & 0.013 & 0.013 & 0.028 \\
\hline
\end{tabular}

Model 1: non-adjusted. Model 2: adjusted for sex, age. Model 3: further adjusted for smoking, alcohol. Model 4: further adjusted for HDL-C, LDL-C, DM.

$\mathrm{CHD}$, coronary heart disease; $\mathrm{PP}$, pulse pressure.

\section{DISCUSSION}

In this cross-sectional study, we found the significant association between high PP and CHD in Korean elderly using data from KNHANES VII. As the previous studies, PP increased with age $[8,12]$ and high PP was positively correlated with the incidence of $\mathrm{CHD}$ and related with higher SBP, lower DBP and higher FBG. Also, the association between PP and the incidence of CHD was consistent after adjustment for age, sex, smoking habits, alcohol consumption, serum HDL-C, LDL-C and DM. Previous studies have reported that high PP is an important component of the CHD risk. In the Framingham Heart Study, it was confirmed that high PP, as well as $\mathrm{SBP}$ and $\mathrm{DBP}$, is a risk factors for CHD [13]. Assmann et al. [9] suggested that high PP is an independent determinant of CHD risk, even in those with normal pulse pressure. Glasser et al. [8] confirmed PP is related with incident $\mathrm{CHD}$ with no racial or regional differ- ences in black and white. Selvaraj et al. [12] reported the prognostic value of $\mathrm{PP}$ and its association with multiple adverse cardiovascular outcomes.

However, in the study comparing central, and peripheral SBP and PP in Asian population, central SBP is more valuable than other blood pressure parameters for prediction of cardiovascular mortality [14]. In addition, though PP is identified as an independent risk factor for CHD, it is still controversial whether it should be the target of antihypertensive therapy [15].

PP is composed of various factors such as arterial stiffness, ventricular ejection, and timing of wave reflection. Among these, aging has a great influence on arterial stiffness which results in increased SBP and decreased DBP. The increased systolic afterload causes left ventricular hypertrophy (LVH) and left ventricular oxygen requirement [16]. Also, due to abnormalities in iso-volumetric relaxation, $\mathrm{LVH}$ increases the systolic period while decreasing the diastolic period [17]. In 
this study, we confirmed positive correlation between age and $\mathrm{PP}$ and $\mathrm{CHD}$, which is considered to be such a mechanism.

In this observational study, we found PP is positively related with high FBG. FBG is one of the diagnostic criteria for DM. It is well known that DM is a high risk factor for cardiovascular disease. In the study on arterial stiffness and glucose tolerance, poor glucose tolerance status was correlated with increased arterial stiffness and decreased arterial compliance even before the onset of DM [18]. The vessel wall is composed of collagen and elastin, which are influenced by hemodynamic forces and extrinsic factors including hormone, salt and glucose regulation [16-18]. As impaired glucose tolerance affects arterial stiffness, it is considered FBG and PP are in positive correlation.

The limitations of this study are as follows. First, we did not correct the possible impacts of hypertension on CHD. No investigation of the history of HTN drug treatment and subgroup analysis of the HTN and normotensive group was performed. Also, we did not consider other comorbidities (such as hyperthyroidism) which could affect blood pressure. Second, since this study is a cross-sectional study, we evaluated the association between variables, but it was difficult to explain an exact causal relationship or a predecessor relationship. Third, though we attempted to reduce the effects of confounders bias through by adjustment of covariates, some residual confounding may remain.

Despites these limitations, this study is meaningful in that it used large-scale, nationally-representative database to describe the association between $\mathrm{pp}$ and $\mathrm{CHD}$ in the Korean elderly population. Furthermore, we confirmed the importance of PP which can be easily used in daily clinical practice with recommendation to physicians to consider $\mathrm{PP}$ in addition to SBP and DBP.

In this study, we found the significant association between $\mathrm{PP}$ and CHD in Korean elderly. Our findings suggest the usefulness of clinical data of PP which are non-invasive and has easy clinical applicability in primary care settings. A prospective study on the association between $\mathrm{PP}$ and $\mathrm{CHD}$ in Korean elderly is needed.

\section{REFERENCES}

1. Kalra A, Bhatt DL, Rajagopalan S, Suri K, Mishra S, Iqbal $\mathrm{R}$, et al. Overview of coronary heart disease risk initiatives in South Asia. Curr Atheroscler Rep 2017;19:25.

2. Sanchis-Gomar F, Perez-Quilis C, Leischik R, Lucia A. Epidemiology of coronary heart disease and acute coronary syndrome. Ann Transl Med 2016;4:256.

3. Ford ES, Capewell S. Proportion of the decline in cardiovascular mortality disease due to prevention versus treatment: public health versus clinical care. Annu Rev Public Health 2011;32:5-22.

4. Townsend N, Wilson L, Bhatnagar P, Wickramasinghe K, Rayner M, Nichols M. Cardiovascular disease in Europe: epidemiological update 2016. Eur Heart J 2016;37:3232-45.

5. Mokdad AH, Mensah GA, Krish V, Glenn SD, Miller-Petrie MK, Lopez AD, et al. Global, regional, national, and subnational big data to inform health equity research: perspectives from the Global Burden of Disease Study 2017. Ethn Dis 2019;29(Suppl 1):159-72.

6. Poulter N. Coronary heart disease is a multifactorial disease. Am J Hypertens 1999;12(10 Pt 2):92S-5S.

7. Dalen JE, Alpert JS, Goldberg RJ, Weinstein RS. The epidemic of the 20(th) century: coronary heart disease. Am J Med 2014;127:807-12.

8. Glasser SP, Halberg DL, Sands C, Gamboa CM, Muntner $\mathrm{P}$, Safford M. Is pulse pressure an independent risk factor for incident acute coronary heart disease events? The REGARDS study. Am J Hypertens 2014;27:555-63.

9. Assmann G, Cullen P, Evers T, Petzinna D, Schulte H. Importance of arterial pulse pressure as a predictor of coronary heart disease risk in PROCAM. Eur Heart J 2005;26:2120-6.

10. Stevenson LW, Perloff JK. The limited reliability of physical signs for estimating hemodynamics in chronic heart failure. JAMA 1989;261:884-8.

11. Task Force for the management of arterial hypertension of the European Society of Hypertension; Task Force for the management of arterial hypertension of the European Society of Cardiology. 2013 ESH/ESC guidelines for the management of arterial hypertension. Blood Press 2013;22:193-278.

12. Selvaraj S, Steg PG, Elbez Y, Sorbets E, Feldman LJ, Eagle $\mathrm{KA}$, et al.; REACH Registry Investigators. Pulse pressure and risk for cardiovascular events in patients with atherothrombosis: from the REACH registry. J Am Coll Cardiol 2016;67:392403.

13. Franklin SS, Khan SA, Wong ND, Larson MG, Levy D. Is pulse pressure useful in predicting risk for coronary heart disease? The Framingham heart study. Circulation 1999;100:35460.

14. Wang KL, Cheng HM, Chuang SY, Spurgeon HA, Ting CT, Lakatta EG, et al. Central or peripheral systolic or pulse pressure: which best relates to target organs and future mortality? J Hypertens 2009;27:461-7.

15. Strandberg TE, Pitkala K. What is the most important com- 
ponent of blood pressure: systolic, diastolic or pulse pressure? Curr Opin Nephrol Hypertens 2003;12:293-7.

16. Mitchell GF. Arterial stiffness and hypertension. Hypertension 2014;64:13-8.

17. Veerasamy M, Ford GA, Neely D, Bagnall A, MacGowan G, Das R, et al. Association of aging, arterial stiffness, and car- diovascular disease: a review. Cardiol Rev 2014;22:223-32.

18. Henry RM, Kostense PJ, Spijkerman AM, Dekker JM, Nijpels G, Heine RJ, et al.; Hoorn Study. Arterial stiffness increases with deteriorating glucose tolerance status: the Hoorn Study. Circulation 2003;107:2089-95. 